\title{
Topography and Source Analysis of Brain Activity Associated with Selective Spatial Aftention and Memory Search
}

\author{
A.A. Wijers*, G. Mulder*, H. van Hooff*, J. Lange*, M.J. Peters*, and Z. Dunajski*
}

Summary. We investigated the topographical aspects of the ERP reflections of visual spatial attention and memory search. Spatial attention was found to enhance the amplitudes of the P1 and N1 deflections. The brain activity in the P1-N1 latency range could be modeled with a single moving equivalent dipole, or alternatively with two stationary dipoles in a spatio-temporal dipole model. The dipoles were located in mesial and lateral posterior brain regions. Similar dipole solutions were obtained for ERPs to attended and unattended stimuli. Increasing the memory search requirements of the task resulted in an increase of late negativity, which was topographically distinguishable from the P3 component.

Key words: Visual spatial attention; Memory search; P1; N1; P3; Search-related negativity.

\section{Introduction}

A vast amount of information reaches the brain from the sense organs. Obviously the brain can not process all of this information to the same extent. Selectivity of processing is a central aspect of human cognition; it is regarded as the major topic in the study of attention. It has proven extremely useful to study selective attention with the aid of ERPs. In ERP research, the tasks typically consist of rapidly presented series of stimuli, which differ randomly from trial to trial with respect to simple physical selection attributes, like, in the visual modality, spatial location (e.g., right versus left of fixation), color (e.g., red versus blue), etc. Subjects are instructed to attend to one of the stimulus categories only, in order to detect the low-probability occurrence of target stimuli within the to-be attended category.

The timing and morphology of the effects of attention on the ERPs depend on input-modality and (at least in the visual modality) the type of selection attribute; this paper is concerned with visual selections on the basis of spatial location. Spatial attention typically results in ERPs with an enhanced series of positive and negative

*Experimental and Occupational Psychology, University of Groningen, Groningen, The Netherlands and Faculty of Applied Physics, University of Twente, Enschede, The Netherlands.

Accepted for publication: March 8, 1993.

Correspondence and reprint requests should be addressed to Dr. A.A. Wijers, Experimental and Occupational Psychology, University of Groningen, Grote Kryisstraat2/1,9718 TSGroningen, The Netherlands.

Copyright $\odot 1993$ Human Sciences Press, Inc. deflections at posterior electrodes, most consistently so for the P1 (latency about 100-150 ms) and N1 (latency about 170-220 ms) components. These effects have been interpreted as reflecting a modulation of the exogenous P1 and N1 components, indicative of attentional control over early perceptual analysis. Such a 'sensory gain' hypothesis of spatial attention predicts that the same generators should underlie the ERPs to both attended and unattended stimuli. Evidence supportive of this prediction has recently been reported (e.g, Mangun et al. 1993).

In previous research we aimed to investigate the selectivity of working memory operations by measuring ERPs in 'selective search tasks'. The stimuli were letters varying with respect to simple visual attributes (e.g. color, Wijers et al. 1989a). Subjects were instructed to discriminate the stimuli on the basis of this attribute, to attend to one stimulus category only and to search for the occurrence of target letters within that category. Target letters were members of a pre memorized memory set containing a variable number of letters. As usual, a Pz maximal positivity ('P3') was elicited by the relevant target stimuli, but was absent in the ERPs to irrelevant target stimuli. Increasing the number of letters in the memory set ('memory load') resulted in a decrease of P3 amplitude, as had been shown previously for search paradigms not involving a selective attention manipulation (e.g., Brookhuis et al. 1981). It was argued that this reduction resulted at least in part from an increase of a late (onset at about $250-300 \mathrm{~ms}$ ), prolonged negativity as a function of memory load, overlapping the P3. This 
'search-related negativity' was also present in the ERPs to non target letters in the to-be-attended category, which did not show a P3 component, but it was completely absent in the ERPs evoked by unattended target and non target letters. It was argued that the P3 and searchnegativity are distinct ERP components. The negativity was thought to be the manifestation of a controlled (i.e., an attention demanding) search operation in working memory. This working memory process could apparently efficiently be restricted to the selected category of stimulus letters.

In the present experiment we aimed at a sourceanalysis of the P1 and N1 components in a spatial selective search task, and at a more extensive comparison of the topographical characteristics of the P3 and search negativity.

\section{Methods}

\section{Stimuli}

Four subjects received series of 240 letters presented at the horizontal meridian, randomly to the left or right (each with a probability of .5) of a central fixation-dot. Stimulus duration was $35 \mathrm{~ms}$ and the inter stimulus intervals were randomly chosen as 700,800 , or $900 \mathrm{~ms}$. Each series was preceded by a new set of either 1 or 4 memory set letters (targets) and a cue indicating whether the left or right visual field letters were to-be- attended. Subjects were instructed to detect the target letters at the attended side, and to respond to those stimuli only with a finger-lift response of the preferred hand. In 1/4 of both the left and right side presentations a target letter was presented. The height of the letters was 1.5 degree, and the distance between the inner edge of the letters and fixation was 2.3 degree. Subjects received 6 stimulus blocks, of which two were with a memory load of 1 and four with a load of 4 . In half of the blocks they attended to the right side and in half to the left side. These conditions were counterbalanced within and between subjects.

\section{Recording}

The EEG was recorded from in total 32 electrode positions (recorded in two separate sessions of 16 channels). These were the positions of the 10-20 system and intermediate positions. The signals were digitized with a sample rate of $100 \mathrm{~Hz}$ (band pass $.05-35 \mathrm{~Hz}$ ). The signals were referred to the right mastoid. The EOG was recorded from electrodes above and aside the right eye.

\section{Data analysis}

The ERPs were averaged off line. Trials containing EOG artifact ( $>100 \mu$ Volt) or incorrect responses were rejected. The ERPs were aligned to a $100 \mathrm{~ms}$ pre stimulus baseline. P1 and N1 latencies and amplitudes were measured at T5a (halfway between $\mathrm{O} 1$ and T5) and T6a. These measures, and the behavioral measures (RT and errors) were submitted to SPSS MANOVAs. For equivalent dipole fitting we used a program developed by de Munck (1989). The volume-conductor was modeled with four concentric spheres (brain, brain-fluid, skull, and scalp) with radii of respectively $.85, .88, .92$, and 1.0. The skull was modeled as anisotropic, with the conductivity in the radial direction a factor of ten lower than in the tangential direction (see Peters and de Munck 1990). As a model of the source, we used both a moving dipole model, in which a single equivalent dipole (ECD) was fitted to the data at individual sample points, and a spatio-temporal dipole model. The spatio-temporal model localized the activity pattern of a (small number of) fixed dipole(s) in a certain latency range. Sourceanalysis was applied to the grand-average wave forms and the results will be reported for the memory load 1 condition only; however, very similar results were obtained for the memory load 4 condition.

\section{Results}

\section{Performance}

Mean reaction-time was faster in the memory load 1 condition than in the memory load 4 condition $(495 \mathrm{~ms}$ versus $590 \mathrm{~ms}, \mathrm{~F}(1,3)=56.96, \mathrm{p}<.05)$. Less relevant targets were missed in the memory load 1 condition than in the memory load 4 condition $(0.17 \%$ versus $4 \%, F(1,3)=25.5$, $\mathrm{p}<.05)$.

\section{ERPs - P1 and N1}

The earliest visible peaks in the ERPs were the posterior P1 and N1 components. Table 1 shows the mean latencies and amplitudes of the P1 and N1 components at T5a and T6a. Both P1 and N1 latencies were shorter at the electrode contralateral to the visual field of stimulus presentation. P1 amplitude was larger at the ipsilateral electrode, whereas N1 amplitude was larger at the contralateral electrode. Attention enhanced P1 and N1 amplitudes, but this effect was much more pronounced over the left hemisphere than over the right hemisphere. There was a significant main effect of attention for P1 amplitude $(F(1,3)=10.59, p<.05)$ and an attention by hemisphere interaction for both $\mathrm{P} 1$ amplitude $(\mathrm{F}(1,3)=52.41, \mathrm{p}<.01)$ and $\mathrm{N} 1$ amplitude $(\mathrm{F}(1,3)=59.54$, $\mathrm{p}<.01$ ).

The iso-contour maps in the latency range of the maximal P1 amplitude (150-170 ms) all showed a clear maximum over the ipsilateral posterior scalp, with negativity 
Table 1

\begin{tabular}{|c|c|c|c|c|}
\hline \multicolumn{5}{|c|}{ P1 latency } \\
\hline & T5a & T6a & & \\
\hline Left Visual Field & 171 & 155 & & \\
\hline Right Visual Field & 140 & 176 & & \\
\hline \multicolumn{5}{|c|}{ N1 latency } \\
\hline & T5a & T6a & & \\
\hline Left Visual Field & 212 & 202 & & \\
\hline \multirow[t]{4}{*}{ Right Visual Field } & 195 & 227 & & \\
\hline & \multicolumn{4}{|c|}{ P1 amplitude } \\
\hline & \multicolumn{2}{|c|}{ T5a } & \multicolumn{2}{|c|}{ T6a } \\
\hline & att. & unatt. & att. & unatt. \\
\hline Left Visual Field & 5.42 & 2.07 & 1.53 & 0.96 \\
\hline \multirow[t]{4}{*}{ Right Visual Field } & 2.65 & 0.22 & 2.30 & 2.17 \\
\hline & \multicolumn{4}{|c|}{ N1 amplitude } \\
\hline & \multicolumn{2}{|c|}{ T5a } & \multicolumn{2}{|c|}{ T6a } \\
\hline & att. & unatt. & att. & unatt. \\
\hline Left Visual Field & -2.08 & 0.13 & -4.47 & -4.52 \\
\hline Right Visual Field & -7.51 & -5.20 & -1.73 & -1.00 \\
\hline
\end{tabular}

over the contralateral and anterior regions. Before this latency, the maps didn't show a consistent pattern. Contour maps later than $170 \mathrm{~m}$ showed an increase of contralateral negativity, whereas the positivity diminished and shifted more anteriorly, reflecting the evolution of the contralateral N1 component.

Source-analyses with the moving dipole model gave satisfactory solutions for the latency range of about 150$210 \mathrm{~ms}$. In this latency range the dipole systematically moved from sample to sample from a medial occipitalparietal location to more lateral, occipital-parietal-temporal locations, contralateral to the visual field of stimulation (see figure 1). Very comparable dipole solutions were obtained for the ERPs elicited by attended and unattended stimuli.

The same data could be fitted with a spatio-temporal dipole model, which explained the ERP activity in the 90-220 (230) latency range with two stationary dipoles, one of which was located at medial parieto-occipital areas, and the other at lateral occipito-parieto-temporal areas, contralateral to the visual field of stimulation (table 2). The temporal functions of activation strength for each dipole over the course of the analyzed latency interval, showed that in all cases the medial dipole reached maximal activation earlier than the lateral dipole. Again, very comparable solutions were obtained for the attended and unattended stimuli.
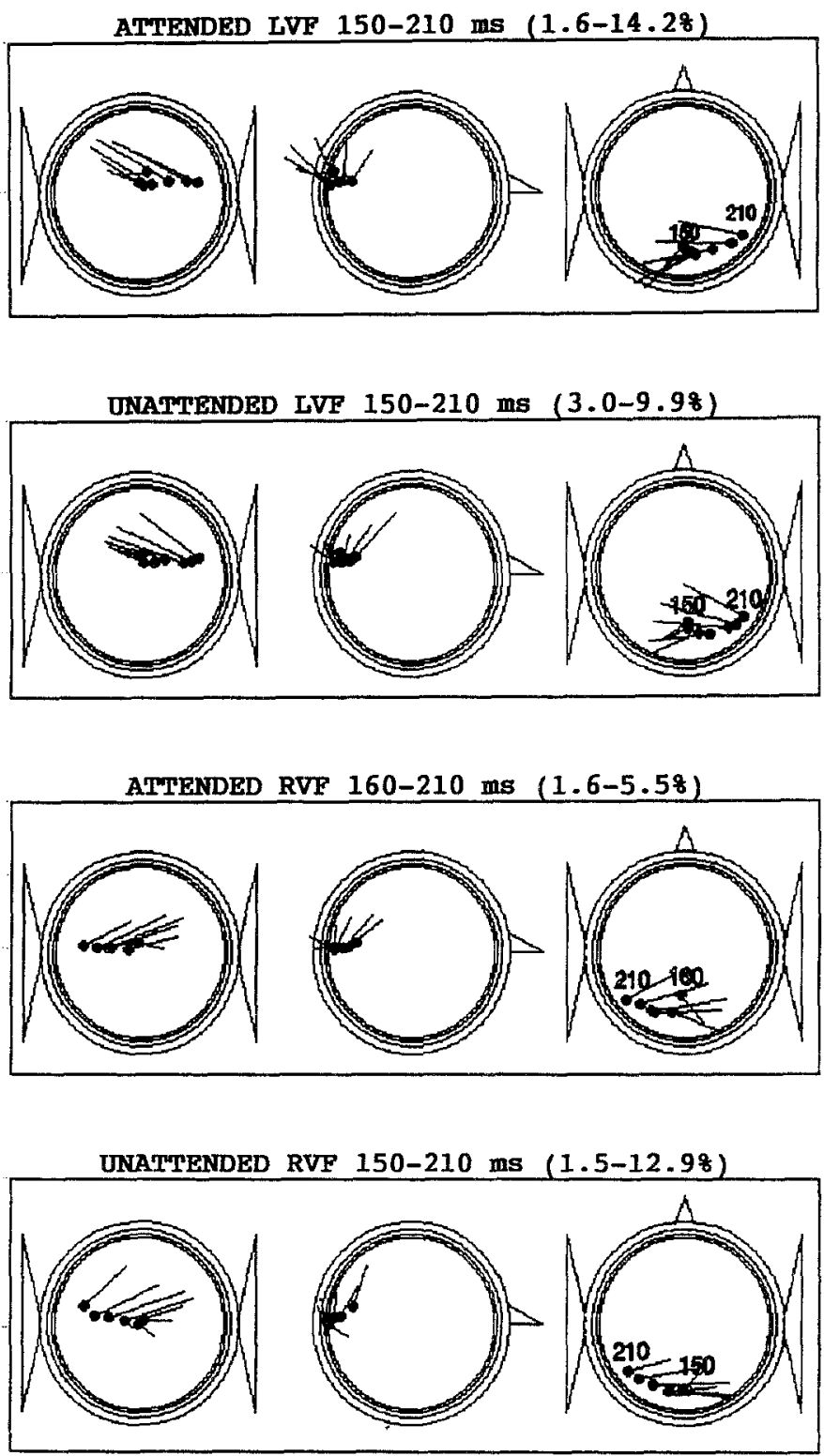

Figure 1. Results of source-analysis (moving dipole model) for the non target stimuli in the memory load 1 condition. Left to right shows back view, lateral view and top view, respectively. Dipole solutions are shown for individual sample-points (10 ms intervals). The range of residual variances for each stimulus category is shown in parentheses. LVF=stimulus presentation in left visual field. RVF=right visual field.

\section{P3 and search negativity}

As had been observed previously, when target letters were presented at the to-be-attended stimulus position, the ERPs evoked by these targets showed a large posterior positivity (P3). No such positivity was observed when target letters were presented at the unattended position. With an increase of memory load from 
Table 2. Results of spatio-temporal source-analysis for non target stimuli in the memory load 1 condition. LVF=left visual field, RVF=right visual field. RAD=radius of the location of the ECD expressed as the proportion from the radius of the sphere model of the head (RAD=0 is in the centre of the sphere). THETA=angle between the radius of the ECD-location and the z-axis running from the middle of the sphere through $\mathrm{Cz}$. PHI=angle between the projection of the ECD location in the xy-plane and the x-axis running from the nasion to the inion. PHI starts at zero at the inion, and is negative for the left side of the head and positive for the right. THETAI and PHIl are the orientation parameters in local spherical coordinates (THETA $1=0$ corresponds to a purely radial dipole; THETA $1=90$ corresponds to a purely tangential dipole).

\begin{tabular}{|c|c|c|c|c|c||}
\hline \multicolumn{7}{|c||}{ LVF attended (90-230 ms) } \\
\hline DIP & RAD & THETA & PHI & TH1 & PHI1 \\
1 & .68 & 73.8 & -8.0 & 15.7 & -108.2 \\
2 & .85 & 76.6 & 40.35 & 59.1 & 26.0 \\
\hline \multicolumn{7}{|c||}{} \\
\hline DIP & RAD & THETA & PHI & TH1 & PHI1 \\
1 & .65 & 67.0 & -11.6 & 27.3 & -11.1 \\
2 & .85 & 76.4 & 39.7 & 48.8 & 21.5 \\
\hline \multicolumn{6}{|c||}{} \\
\hline DIP & RAD & THETA & PHI & TH1 & PHI1 \\
1 & .53 & 72.0 & 12.72 & 31.5 & 44.4 \\
2 & .83 & 85.8 & -28.4 & 63.28 & -52.46 \\
\hline \multicolumn{7}{|c|}{} \\
\hline \multicolumn{7}{|c|}{ RIP } & RAD & THETA & PHI & TH1 & PHI1 \\
1 & .67 & 78.6 & -10.7 & 16.8 & 135.1 \\
2 & .85 & 86.2 & -29.7 & 30.5 & -43.3 \\
\hline \multicolumn{7}{|c}{} \\
\hline
\end{tabular}
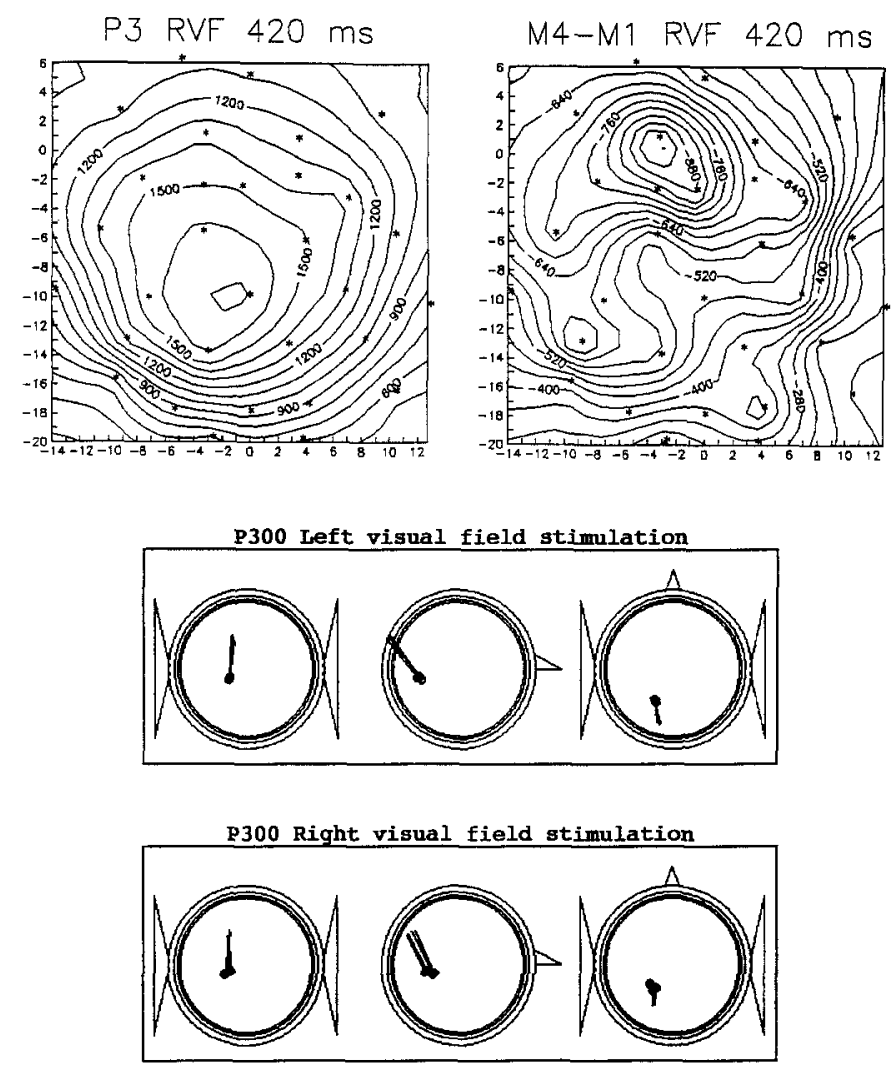

Figure 2. Upper panel: Iso-contour maps at $420 \mathrm{~ms}$ for stimuli presented in the right visual field. The left panel shows the topography of the P3 component (ERPs to attended target stimuli) and the right panel shows the search related negativity (subtraction potential of the ERPs to attended non targets in the memory load 4 condition minus the ERPs to attended non targets in the memory load 1 condition). The maps show a top view in which the $y$-axis corresponds to the anterior-posterior direction (- is posterior). $C z$ is at $x=0, y=2.5$. Lower Panel: Results of source analysis for the grand-average ERPs to attended target stimuli with the moving dipole approach (sample points in the 400-450 ms interval: P3).

Table 3: Results of spatio-temporal source-analysis for the attended target stimuli in the memory load 1 condition, separately for the attend-right and attend left conditions.

\begin{tabular}{||c|c|c|c|c|c|c|}
\hline Condition & RAD & THETA & PHI & TH1 & PHI1 & Res. \\
Attend-L & .59 & 103.8 & -23.2 & 77.7 & 165.6 & $1.6 \%$ \\
Attend-R & .48 & 103.5 & -32.2 & 87.0 & 167.8 & $1.6 \%$ \\
\hline
\end{tabular}


1 to 4 letters, the ERPs showed increasing late negativity (latency range about $300-550 \mathrm{~ms}$ ); this effect was found for both the attended non targets and targets but was absent for the unattended stimuli. A comparison of the scalp topographies of the P3 and the search-related negativity revealed clear differences. In the memory load 1 condition, the $\mathrm{P} 3$ peaked at about $420 \mathrm{~ms}$. In a rather broad latency interval (at least in the interval 400$500 \mathrm{~ms}$ ) the scalp-topography remained very stable and consisted of a positivity at all measured electrode positions, with a clear maximum at the posterior scalp, slightly to the left of the midline (see figure 2). This was the case independent of the visual field in which the to-beattended target letters were presented.

The scalp-topography of the memory load effect was more complicated, and showed more variability within this latency interval. However, whereas the P3 had a maximum at parietal areas, the search negativity was clearly dominant over centro-frontal areas. As for the P3, the search negativity was larger over the left than over the right hemisphere.

A single source model accounted very well for P3 scalp-topography, explaining about $98 \%$ percent of the variance in the $400-450 \mathrm{~ms}$ interval. Very similar ECDs, located in an occipital-temporal region of the left hemisphere, were computed for the attend-right and attendleft conditions (see table 3). The spatio-temporal model and the moving dipole model yielded almost identical results. The moving dipole solution computed an ECD which remained very constant from sample to sample in the latency range of the $\mathrm{P} 3$ peak, explaining between 98.2 and $98.8 \%$ of the variance (see figure 2). Source analysis of the search-related negativity, on the other hand, did not yield any consistent result.

\section{Discussion}

The observed ERP morphology and its modulation by spatial attention in general resembled the results of previous research with this type of paradigm (e.g., Mangun et al. 1993). The earliest visible deflections in this experiment were the P1 and N1 components, both showing prolonged latencies over the ipsilateral hemisphere as compared to over the contralateral hemisphere, which is a common observation (e.g., Rugg et al. 1987). P1 amplitude showed an ipsilateral hemispheric lateralization, and the $\mathrm{N1}$ a contralateral lateralization; this is also a usual finding (e.g., Rugg et al. 1987; Wijers et al. 1989b).

As was expected, both the P1 and N1 were enhanced by spatial attention. This enhancement was found to be more prominent over the left hemisphere; a similar observation was done by Proverbio and Mangun (1992). Both the P1 and N1 showed maxima over the lateral occipital scalp (at electrodes T5a and T6a), about $5 \mathrm{~cm}$ lateral to the mid-sagittal plane. In accordance with a sensory gain hypothesis of spatial attention, and consistent with conclusions reached by Mangun et al. (1993), similar dipole configurations were found to account for the brain responses to attended and unattended stimuli. Mangun et al. (1993) related their findings to a neurophysiological model of vision (as reviewed by Desimone and Ungerleider 1989). On the basis of the current source density distributions of the P1 and N1 components (the P1 maximum lying ventro laterally to the N1 maximum), Mangun et al. (1993) suggested that the P1 and N1 components reflect activity from respectively the 'ventral' and 'dorsal' projection routes. The ventral system, projecting from striate cortex to inferior temporal cortex is important for objects perception (the 'what' system), whereas the dorsal system, projecting from striate cortex to posterior parietal cortex, is primarily engaged in spatial perception (the 'where' system). Our results show a rather different picture. ERP activity in the P1-N1 latency range could be modeled with sources of activity in the posterior brain, moving from a position close to the midline to more lateral areas, or alternatively with two stationary dipoles, the one with an earlier activation maximum located at a medial position, and a second one, with a later activation maximum, at a more lateral position. Thus, surprisingly, these results are more consistent with localization of the N1 generator in ventral areas. The medial dipoles were oriented so that they pointed to the hemisphere ipsilateral to the visual field of stimulation. This is reminiscent of the explanation proposed for the 'paradoxical lateralization' of the P1 evoked by reversing checkerboard stimulation (Barrett et al. 1976); here the P1 was assumed to be of striate origin. Therefore, one could suggest that spatial attention modulates activity of the primary visual cortex as well as the information flow into ventral regions. However, the latency of our P1 component is probably too late to reflect striate activity; Mangun et al. (1993) have found an earlier NP60 component, presumably of striate origin, to precede the P1 in a spatial attention task. In addition, the location of the medial ('P1') ECDs is probably dorsal to the primary visual area. In our coordinate system, the location of the medial dipoles would lie beneath a position approximately halfway between $\mathrm{Pz}$ and $\mathrm{Oz}$, whereas the calcarine sulcus is reported to lie approximately beneath $\mathrm{Oz}$ (Steinmetz et al. 1989). One could speculate, therefore, that the $\mathrm{P} 1$ and not the N1 reflects activity along the dorsal projection route.

The topographical data provided clear evidence for the idea that working memory operations are manifested by a prolonged negativity which is to be distinguished from the $\mathrm{P} 3$ component. Although we are aware that there is accumulating evidence that the $\mathrm{P} 3$ represents the compound activity of multiple generators (e.g., Kropotov 
and Ponomarev 1991), in this experiment P3 topography could consistently (over conditions and over samplepoints) be modeled with a single ECD in the left posterior (occipital-temporal) hemisphere. Left-hemispheric lateralization of the $\mathrm{P} 3$ is to our knowledge not a wellknown phenomenon. It could be that the lateralization of the $\mathrm{P} 3$ and the particular sources that contribute most importantly to the scalp-recorded P3, are task-specific. The search-related negativity was clearly more anteriorly distributed than the P3 and showed a complex topography, suggesting the involvement of multiple generators. This could indicate that the working memory system involves a connected network of different brain regions.

\section{References}

Barrett, G., Blumhardt, L., Halliday, A.M., Halliday, E. and Kriss, A. A paradox in the lateralization of the visual evoked response. Science, 1976, 261: 253-255.

Brookhuis, K.A., Mulder, G., Mulder, L.J.M., Gloerich, A.B.M., van Dellen, H.J., van der Meere, J.J. and Ellerman, H.H. Late positive components and stimulus evaluation time. Biological Psychology, 1981, 13: 107-123.

De Munck, J.C. A mathematical and physical interpretation of the electromagnetic field of the brain. Doctoral Thesis. 1989.

Desimone, R. and Ungerleider, L.G. Neural mechanisms of visual processing in monkeys. In: F. Boller and J. Grafman (Eds.), Handbook of Neuropsychology, Vol. 2 (pp. 267-298).
Elsevier, Amsterdam, 1989: 267-298.

Kropotov, J.D. and Ponomarev, V.A. Subcortical neuronal correlates of component $\mathrm{P} 300$ in man. Electroencephalography and clinical Neurophysiology, 1991, 78: 40-49.

Mangun, G.R., Hillyard, S.A. and Luck, S.J. Electrocortical substrates of visual selective attention. In: S. Kornblum and D.E. Meyer (Eds.), Attention and Performance XIV, Erlbaum, Hillsdale NJ, 1993: in press.

Peters, M. and de Munck, J. In: F. Grandori, M. Hoke and G.L. Romani (Eds.), Advances in Audiology vol. 6, Auditory Evoked Magnetic Fields and Electric Potentials. Karger, Basel, 1990: 70-102.

Proverbio, A.M. and Mangun, G.R. Right and left hemisphere roles for attention: electrophysiological evidence. Poster presented at EPIC X, 1992.

Rugg, M.D., Milner, A.D., Lines, C.R. and Phalp, R. Modulation of visual event-related potentials by spatial and non-spatial visual selective attention. Neuropsychologia, 1987, 25: 85-96.

Steinmetz, H., Frst, G. and Meyer, B. Craniocerebral topography within the international 10-20 system. Electroencephalography and clinical Neurophysiology, 1989, 72: 499-506.

Wijers, A.A., Lamain, W., Slopsema, S., Mulder, G. and Mulder, L.J.M. An electrophysiological investigation of the spatial distribution of attention to colored stimuli in focused and divided attention conditions. Biological Psychology, 1989b, 29: 213-245.

Wijers, A.A., Mulder, G.,Okita, T., Mulder, L.J.M. and Scheffers, M.K. Attention to colour: An ERP-analysis of selection, controlled search and motor activation. Psychophysiology, 1989a, 26: 89-109. 